\title{
Relación de la carga de entrenamiento con las emociones y el rendimiento en baloncesto formativo Relation of training load with emotions and performance in formative basketball
}

\author{
*Victor Hugo Duque Ramos, **María Reina Román, **David ManchaTriguero, **Sergio José Ibáñez Godoy, \\ $* * *$ Pedro Saenz-Lopez Buñuel \\ *U niversidad de Antioquia (Colombia); **U niversidad de Extremadura; ***U niversidad de Huelva
}

\begin{abstract}
Resumen. El objetivo del estudio fue identificar las relaciones entre carga de entrenamiento con variables emocionales y de rendimiento en baloncesto formativo. Los participantes fueron 82 jugadores y jugadoras (40 hombres y 42 mujeres) de categorías cadete ( 15 y 16 años) e infantil (13 y 14 años) con una edad media de 14,6 años. Se midió la carga interna con la FrecuenciaC ardiaca M áxima, y la carga externa por medio de acelerometría, lainteligencia emocional, rendimiento percibido y variables relacionadas con el bienestar como la fatigay dolor muscular. Los resultados mostraron que laFC está relacionada con una mayor percepción de rendimiento, así como lacarga externa con la fatiga. La fatiga y el dolor muscular mostraron una fuerte relación entre ellas y, a su vez, con anotar menos puntos, menor percepción de rendimiento y con una regulación emocional negativa. El rendimiento percibido relacionó positivamente con todas las variables de la inteligencia emocional. Con estos datos, los entrenadores disponen de más recursos y conocimientos a la hora de planificar y diseñar las sesiones de entrenamiento lo que repercutirá en el rendimiento por parte de los deportistas. Se recomienda entrenar con intensidad sin llegar a percibir fatiga ni dolor muscular, así como desarrollar las variables de la inteligencia emocional.
\end{abstract}

Palabras clave. deporte escolar, inteligencia emocional, rendimiento percibido, bienestar, fatiga y dolor muscular

\begin{abstract}
The objective of the study was to identify the relationships between training load with emotional and performance variables in formative basketball. The participants were 82 players in the formative categories. Internal load, external load, emotional intelligence, perceived performance, and well-being-related variables such as fatigue and muscle pain weremeasured. The results show that heart rate isrel ated to a higher perception of performance, as well as exter nal load with fatigue. Similarly, fatigue and muscle pain had a strong relationship between them and, in turn, with less score in matches, less perception of performance and negative emotional regulation. The perceived performance relates positively to all the variables of emotional intelligence. W ith these data, the coaches have more resources and knowledge when it comes to planning and design the training sessions, which will improve performance by athletes. It is recommended to train with intensity without perceiving fatigue or muscular pain, as well as developing the variables of emotional intelligence.
\end{abstract}

Key words. school sport, emotional intelligence, perceived performance, wellness, fatigue and muscle pain.

\section{Introducción}

La implementación de estrategias efectivas en el entrenamiento es primordial para promover adaptaciones que mejoren el rendimiento (Hernández, Casamichana \& Sánchez-Sánchez, 2017). Estas estrategias van a depender de una variedad de factores que influyen en las demandas externas, respuestas internas, rendimiento y emociones (A guiar, Botelho, Lago, Maças $\&$ Sampaio, 2012). Para optimizar el entrenamiento, es necesario disponer de recursos suficientes que permitan definir objetivos y controlar la evolución de los jugadores (Reina, M ancha-Triguero, García-Santos,

Fecha recepción: 29-07-20. Fecha de aceptación: 15-10-20 Pedro Saenz López

psaenz@uhu.es
García-Rubio \& Ibáñez, 2019).

En cuanto a la cuantificación de la carga de entrenamiento, la carga externa indica el volumen o la intensidad como estímulo expuesto (Petway, Freitas, Calleja González, Medina Leal \& Alcaraz, 2020), mientras que las respuestas internas representan las reacciones fisiológicas del jugador como resultado del estímulo (Reina, Rubio, Antúnez \& Ibáñez, 2020). En baloncesto, estas demandas y respuestas varían según el puesto del jugador, nivel físico o el estímulo de entrenamiento. Por tanto, la monitorización de estos resultados es necesaria a la hora de prescribir de forma individualizada el entrenamiento. Al hacerlo, podrá ser más efectivo en la medida en que sea específico para un deporte y de intensidad suficiente para proporcionar adaptaciones fa vorables a la vez que se reduce la probabilidad de lesión (Reina, GarcíaRubio \& Ibáñez, 2020). 
El uso de sistemas inerciales para el control de las cargas de entrenamiento y competición se encuentra en auge (Fox, Scanlan \& Stanton, 2017). Estos sistemas se componen de microsensores, tanto de acelerómetro como giroscopios y magnetómetros, de esta forma se utilizan para calcular continuamente la posición, dirección y velocidad del jugador en movimiento a través del uso de tecnología UltraW ided Band (UWB), sin necesidad de referencias satélites (Bastida-Castillo, et al. , 2019). Es unaherramientaútil en los deportesindoor, donde la señal a través de Sistemas de Posicionamiento Global (GPS) no es fiable. Por tanto, la aplicación de este tipo de microsensores para la determinación de las demandas externas en baloncesto se centra principalmente en el uso de acelerómetros triaxiales (Fox, et al., 2017). Definir estas demandas externas junto con sistemas de medición de respuestas internas proporcionan información valiosa (Boyd, Ball, Aughey $\&$ performance, 2011; Chambers, Gabbett, Cole\& Beard, 2015). El uso de la frecuencia cardíaca, como medida de control de carga interna para determinar la intensidad del ejercicio, está bastante definida en el mundo del entrenamiento (C astagna, Impellizzeri, Chaouachi, Bordon \& M anzi, 2011; M as, Guzmán, \& Martínez, 2018).

A simismo, valorar la fatiga de los jugadores durante la temporada es necesario para evitar lesiones, pérdidas de rendimiento o la aparición de sobre-entrenamiento (Twist \& Highton, 2013). Actualmente está extendido el uso de diferentes métodos para controlar la fatiga en los deportes de equipo, como los cuestionarios o valoraciones subjetivas. La más usada en el ámbito deportivo es la Ratings of Perceived Exertion (RPE) propuesta por Borg (1962), donde los jugadores señalan al final de cada sesión o partido la intensidad que le ha demandado. En cuanto a valoraciones subjetivas de la fatiga, McLean, Coutts, Kelly, M cG uigan y Cormack (2010) elaboraron un cuestionario de bienestar en el que proponen cinco preguntas relacionadas con la fatiga percibida, la calidad del sueño, el daño muscular, los niveles de estrés y el humor. Esta herramienta ayuda a conocer la evolución de la fatiga en relación con la carga externa, siendo una propuesta basada en el uso de métodos no invasivos y económicos que permiten al cuerpo técnico tener una información válida y fiable (McLean, Coutts, Kelly, McGuigan \& Cormack, 2010).

Uno de estos factores que influyen en estas variables y en diferentes ámbitos de la vida es la Inteligencia Emocional (IE) (Salovey, Mayer, Caruso \& Yoo, 2009). La IE engloba habilidades como: autocontrol, autoconciencia, agilidad mental, la motivación y la con- fianza, que permiten al entrenar tener recursos para ayudar en la identificación y educación de emociones en los jugadores (M ercadet-Portillo \& Inufio-Díaz, 2015).

Se han desarrollado tres categorías principales de instrumentos de IE (M iao, Humphrey \& Q ian, 2017): i) IE basada en el rendimiento a través de la resolución de problemas emocionales con respuestas correctas 0 incorrectas; ii) IE mixta a través de autoinformes en el que los jugadores y jugadoras presentan autopercepcionesindividuales relacionados con las emociones donde indican hasta qué punto están de acuerdo 0 en desacuerdo los ítems; y iii) Autoinforme de las pruebas de habilidad de IE en las que los participantes reciben declaraciones descriptivas e indican hasta qué punto están de acuerdo 0 en desacuerdo con los elementos que son consistentes con la definición de IE de Mayer and Salovey (1997). Con respecto a esta última categoría, es decir, la capacidad de autoinforme de las medidas de IE, uno de losinstrumentos más utilizados eslaWong and Law Emotional Intelligence Scale (W LEIS) deWong \& Law (2002), basada en el modelo de M ayer and Salovey (1997) y ampliamente utilizada (Buchheit, et al., 2013; Campos\& Toscano, 2017).

A pesar de lo analizado, la relación de la IE con el rendimiento deportivo hasido muy poco estudiada, por lo que actualmente se estáconvirtiendo en un constructo de gran interés en el contexto deportivo (M eyer $\&$ Fletcher, 2007), mostrando la influencia que tiene este factor en el éxito o fracaso en el deporte (U phill, Groom \& J ones, 2014). Para el análisis del rendimiento en deportes colectivos han sido comúnmente utilizados los indicadores de rendimiento, principalmente provenientes de las estadísticas de juego (M cGarry, 2009), especialmente en deportes colectivos como el Baloncesto (Ibañez, García-Rubio, Gómez \& Gonzalez-Espinosa, 2018; Ibáñez, García-Rubio, Rodríguez-Serrano \& Feu, 2019). No obstante, en deportes individuales el rendimiento suele determinarse de forma muy precisa, coincidiendo con la marca obtenida por el deportista. Por el contrario, en deportes de equipo, el rendimiento de cada jugador suele ser medido de una forma más superficial e imprecisa, no reflejando realmente la verdadera actuación del individuo dentro de la colectividad (Kais $\&$ Raudsepp, 2004). Por ese motivo, Lourenço,Almagro \& Sáenz-López (2018) han diseñado y validado una escala para evaluar el rendimiento percibido cumplimentada por parte del jugador de forma subjetiva.

O bservando la gran cantidad de parámetros empleados a la hora de estudiar y analizar el entrenamiento deportivo, es necesario comprender cómo la carga tan- 
to interna como externa demandada en los entrena mientos, aspectos relacionados con el bienestar de los deportistas, su inteligencia emocional, así como su percepción de rendimiento se relacionan entre sí (Lourenço, et al., 2018). Del trabajo y la mejora de estos factores dependerá la calidad de los entrenamientos y por ente, el desarrollo individual e integral de los deportistas (Sáenz-López, Ibáñez, Giménez, Sierra \& Sánchez, 2005).

Por tanto, y debido a la escasez de estudios centra dos en categorías de formación y, que además investigan de forma conjunta parámetros de carga, emociones y rendimiento, el principal objetivo de esta investiga ción fue identificar las relaciones entre la carga de entrenamiento que soportan los jugadores durante el entrenamiento con variables emocionales y de rendimiento en baloncesto formativo.

\section{M étodo}

\section{Diseño de la Investigación}

El diseño del presente estudio se encuadra dentro de la investigación correlacional, dónde se estudian las relaciones entre variables con el objetivo de predecir una variable categórica (Thomas, Nelson \& Silverman, 2015).

\section{Muestra}

La muestra estuvo compuesta por 82 jugadores (48,7\% hombres y $51,2 \%$ mujeres) entre 13 y 16 años $(M=14,6)$ pertenecientes a cinco equipos de baloncesto en edad escolar (categoría infantil $43,9 \%$ y cadete $56,1 \%$ ) de un club de la provincia de Huelva (España) inscrito en la liga federadatemporada2018/ 2019. Cada deportista asistió a las tres sesiones de entrenamiento de una hora y media en las que se tomaron los datos. Para llevar a cabo el estudio, los familiares responsables de cada jugador, el club y el cuerpo técnico fueron informados previamente sobre el protocolo de investigación, requisitos, beneficiosy riesgos, y se obtuvo su consentimiento por escrito antes del inicio del estudio, desarrollado en base a las disposiciones éticas de la Decla ración de Helsinki (2013).

\section{Instrumentos}

Para el control de las variables relacionadas con la Carga Interna, el jugador fue equipado con una banda de frecuencia cardíaca GARM IN ${ }^{\mathrm{TM}}$ (Kansas, Estados Unidos) y para el control de las variables relacionadas con la Carga Externa, al deportista se le equipó con un dispositivo inercial W IMUPRO ${ }^{\text {TM }}$ (RealTrack Systems, Almería, Spain) que se colocó en la parte superior de la espalda (zona interescapular) en un chaleco anatómicamente ajustado al cuerpo. Además, se utilizó los softwares SVIVO ${ }^{\text {TM }}$ y SPRO ${ }^{\text {TM }}$ (RealTrack Systems, Almería, España) para el análisis de los resultados (Figura 1).

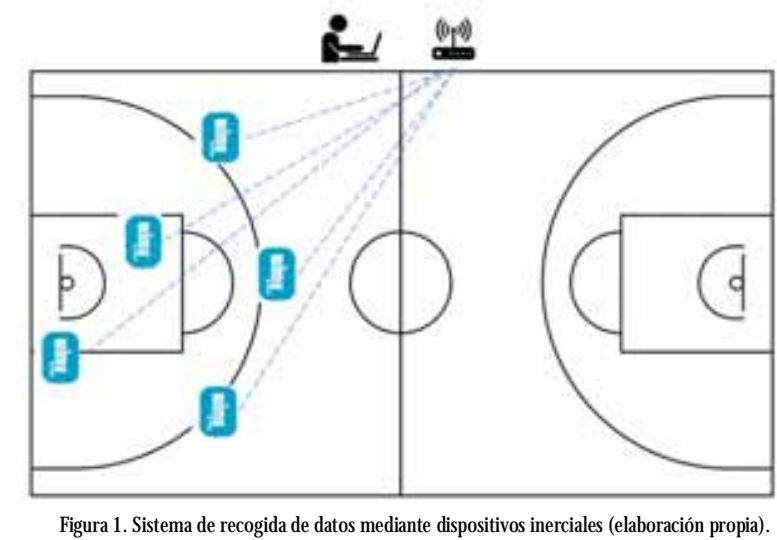

Para el análisis de las variables de Wellness se realizó mediante un Cuestionario de Bienestar, que los jugadores realizaron previo a entrenamientos. En este cuestionario se trataron 5 preguntas relacionadas con la fatiga percibida, la calidad del sueño, el daño muscular, los niveles de estrés y el humor previo al entrenamiento. Cada pregunta se puntúa entre 1- 5, con 1 y 5 representando niveles pobres y muy buenos de bienestar respectivamente (M cLean, et al. , 2010). El grado de «bienestar general» se determina sumando las 5 puntuaciones (Figura 2).

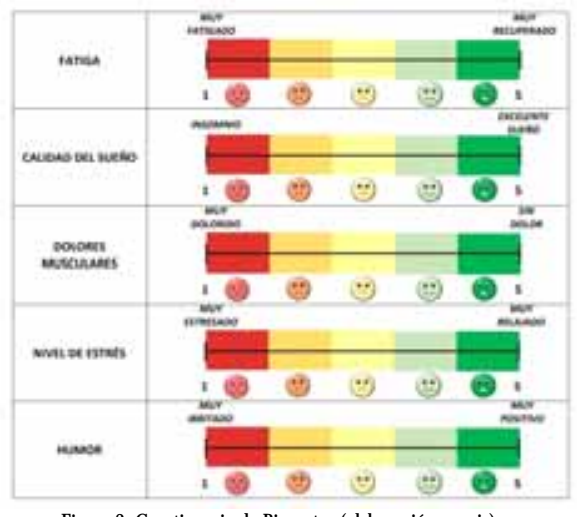

Figura 2. Cuestionario de Bienestar (elaboración propia).

Para el anál isis de las variables emocionales se usó el cuestionario de IE en español W LEIS-S (Pacheco, Rey \& Sánchez-Álvarez, 2019) de su versión en inglés de $\mathrm{Ng}$, Wang, Zalaquett and Bodenhorn (2008), que consta de 16 preguntas clasificadas en evaluación de las propias emociones; evaluación de las emociones de los demás; uso de las emociones; y regulación de las emociones. Las respuestas eran puntuadas en una escala tipo likert 
con un rango de puntuación que oscilaba entre 1 (totalmente en desacuerdo) y 7 (totalmente de acuerdo).

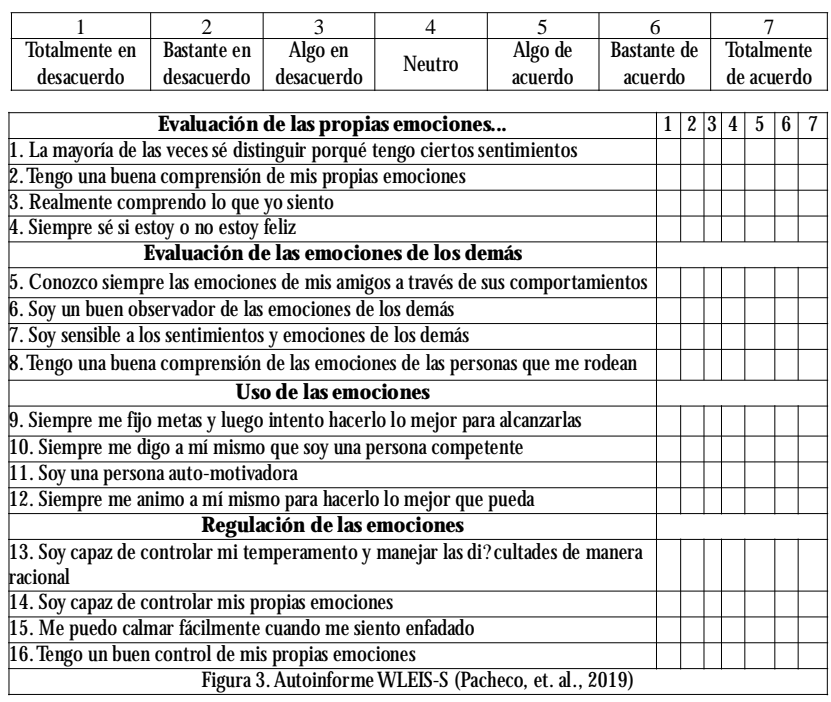

Para el análisis de las variables de Rendimiento se utilizaron las estadísticas de juego determinadas por la Federación Andaluza de Baloncesto en su web. Por otra parte, se utilizó el cuestionario de rendimiento percibido en el deporte de Lourenço, et al. (2018)' , que se compone de 5 preguntas que dan respuesta a la pregunta en general, durante la competición: 1. considero que mi rendimiento está siendo bueno; 2. Estoy satisfecho con mis resultados en la competición; 3. Siento que estoy colaborando, con mi effuerzo y mis actuaciones en competición, a mejorar el nivel competitivo del club o del equipo; 4. Siento que lo estoy haciendo muy bien en la competición; 5. Estoy ofreciendo un buen rendimiento. Las respuestas eran puntuadas en una escala tipo likert con un rango de puntuación que oscilaba entre 1 (totalmente en desacuerdo) y 5 (totalmente de acuerdo) (Figura 4).

\begin{tabular}{|c|c|c|c|c|}
\hline 1 & 2 & 3 & 4 & 5 \\
\hline $\begin{array}{c}\text { Totalmente en } \\
\text { desacuerdo }\end{array}$ & $\begin{array}{c}\text { Algo en } \\
\text { desacuerdo }\end{array}$ & Neutro & Algo de acuerdo & $\begin{array}{c}\text { Total mente de } \\
\text { acuerdo }\end{array}$ \\
\hline
\end{tabular}

\begin{tabular}{|l|l|l|l|l|l|}
\hline \multicolumn{1}{|c|}{ En general, durante la competición: } & 1 & 2 & 3 & 4 & 5 \\
\hline 1. Considero que mi rendimiento está siendo bueno & & & & & \\
\hline 2. Estoy satisfecho con mis resultados en la competición & & & & & \\
\hline $\begin{array}{l}\text { 3. Siento que estoy colaborando, con mi esfuerzo y mis actuaciones } \\
\text { en competición, a mejorar el nivel competitivo del club o del equipo }\end{array}$ & & & & & \\
\hline 4. Siento que lo estoy haciendo muy bien en la competición & & & & & \\
\hline 5. Estoy ofreciendo un buen rendimiento & & & & & \\
\hline
\end{tabular}

\section{Variables}

Para la evaluación del entrenamiento, se han esta blecido cuatro tipos de variables: I) Variables de Carga; II) Variables Well ness (II) Variables emocionales; y IV) Va riables de Rendimiento.

I) Variables de Carga. Para la evaluación de la carga de entrenamiento de los deportistas, se analizaron las siguientes variables, que se dividen en dos grupos según el origen de las demandas (Ibáñez, Antúnez, PinoOrtega \& García-Rubio, 2018): a) Variables de Carga
Interna y b) Variables de Carga Externa.

a) LaVariable de Carga Interna fue evaluada a través de la frecuencia cardíaca (FC) del deportista. LaFC es un indicador de las demandas que tiene el deportista durante una actividad. Se anal iza el siguiente parámetro: $\%$ de Frecuencia Cardíaca Máxima (\%FCM): Es un indicador de la intensidad del esfuerzo físico-fisiológico del atleta durante la prueba, en este estudio se estableció como el mayor resultado obtenido por el deportista durante todas las sesiones. El valor entonces se obtiene del producto de la Frecuencia Cardiaca Media/ Frecuencia Cardíaca Máxima.

b) La Variable de Carga Externa analiza la carga soportada por el deportista en relación con la fuerza gravitacional. Se analizó lasiguiente variable PlayerLoad: es una magnitud vectorial derivada de datos de acelerometría triaxial que cuantifica el movimiento a alta resolución. Las aceleraciones y desaceleraciones se utilizan para construir una medida acumulativa de la tasa de cambio en la aceleración. Se utiliza una medida acumulativa (PL) y una medida de intensidad (PL · min «1) y luego puede indicar la tasa de estrés a la que los jugadores someten su cuerpo durante un cierto período de tiempo. Como unidad de carga, tiene un grado moderado-alto defiabilidad y validez (Barreira, et al. , 2017; Schelling \& Torres, 2016). Para la normalización de los entrenamientos y equidad en función del tiempo de duración, se calculó la variable PlayerLoad/ minuto que aporta el valor de la variable cada minuto de entrenamiento.

II) Variables Wellness: Cuestionario de Bienestar. Con este Instrumento se realizaron a los jugadores y jugadoras preguntas relacionadas con la fatiga percibida, la calidad del sueño, el daño muscular, los niveles de estrésy el humor previo al entrenamiento. No obstante, para esta investigación solo se tuvieron en cuenta las varia bles relacionadas con la carga acumulada por entrenamientos y partidos, por lo que se analizaron las preguntas relacionadas con:
a) Fatiga.
b) Dolor muscular.

III) Variables Emocionales. Se analizaron cuatro va riables mediante un autoinforme, que fueron:

a) Evaluación de las emociones propias (EEP).

b) Evaluación de las emociones de los demás(EED).

c) U so de las emociones (UE).

d) Regulación de las emociones (RE).

Deestascuatro variables seobtiene unaquinta, dónde se evalúa el Total de la escala de inteligencia emocional (TEIE).

IV) Variables de Rendimiento. Se analizaron dos va- 
riables a partir de las estadísticas de juego de los tres últimos encuentros disputados y éstas fueron:

a) Media de puntos.

b) Media de minutos.

Por último, se estableció una Escala de Rendimiento Percibido por parte de los jugadores y jugadoras durante los encuentros:

c) Escala de Rendimiento Percibido (ERP).

\section{Procedimiento}

En las sesiones de entrenamiento, a medida que llegaba cada deportista se le entregaba el Cuestionario de Bienestar para la valoración del estado de fatiga y recuperación, el cual dominaban previamente y completaban de manera individual. Antes del inicio de la sesión, se les equipaba con una banda de frecuencia cardíaca GARM IN $^{\mathrm{TM}}$ y un dispositivo inercial WIMUPRO ${ }^{\mathrm{TM}}$, que se colocó en la parte posterior del torso superior (zona interescapular) en un chaleco específico hecho a medida y ajustado anatómicamente al cuerpo. Este procedimiento se repitió durante las tres sesiones que se llevaron a cabo por equipo y categoría. Todos los entrenamientos fueron como estaban planeados por el entrenador decadaequipo, entrenamientostipo: cal entamiento, situacionesindividuales, oposición, cooperación-oposición, ningún entrenador hacíamodificaciones en su plan de trabajo para realizar el estudio. Posteriormente, a cada deportista se le envió un enlace el cual direccionaba a un formulario de Google donde estaban las preguntas de los cuestionarios de inteligencia emocional W LEIS-S y rendimiento percibido en el deporte, además de los datos edad, sexo, categoría y años de práctica de baloncesto para ser cumplimentados individualmente por medio de su teléfono móvil, tableta u ordenador antes del siguiente entrenamiento.

\section{Análisis estadístico}

En primer lugar, se ha llevado a cabo un análisis exploratorio mediante las pruebas de asunción de criterios. Se han realizado las pruebas de contraste del supuesto de Normalidad (Prueba de Kolmogorov-Smirnov), de contraste del supuesto de Aleatorización (Prueba de Rachas) y decontraste del supuesto de H omocedasticidad (Prueba de Levene) para establecer el modelo de contrastación de la hipótesis adecuado (Field, 2009). En este caso el valor de nivel crítico es mayor que el nivel designificación establecido ( $p<.05)$ (Pardo \& Ruiz, 2002) para rechazar la $\mathrm{HO}$ de normalidad de la muestra. LoS niveles designificación obtenidosen cadaunade las pruebas del análisis exploratorio realizado indican que las variables estudiadas no cumplen de forma conjunta los supuestos por lo que el modelo de contraste de la hipótesis fue no paramétrico. En segundo lugar, se ha realizado un análisis descriptivo con medias y desviación típica sobre las variables analizadas. Final mente, el análisis estadístico utilizado para identificar las relaciones entre las variables de rendimiento físico, wellness, emocionales y de rendimiento se empleó el coeficiente de correlación Rho de Spearman. El software utilizado fue SPSS 24.0 (SPSS Inc., Chicago, IL, EE. UU.).

\section{Resultados}

En la tabla 1, se muestran los resultados descriptivos de las variables anal izadas.

\begin{tabular}{lrr}
$\begin{array}{l}\text { Tabla 1. } \\
\text { Resultados descriptivos de las variables analizadas. }\end{array}$ & & \\
\hline & Media & ST \\
\hline \% FCM & 78.65 & 5.64 \\
PlayerLoad & 50.82 & 13.22 \\
PlayerLoad/ min & 5.58 & 10.37 \\
Fatiga & 3.44 & 1.23 \\
Dolor M uscular & 3.40 & 0.97 \\
Media Puntos & 4.55 & 4.00 \\
Media Minutos & 20.45 & 6.41 \\
ERP & 3.89 & 0.68 \\
Evaluación Emociones Propia & 5.58 & 0.86 \\
Evaluación Emociones Demás & 5.37 & 0.91 \\
Uso Emociones & 5.50 & 1.02 \\
Regulación Emociones & 4.76 & 1.23 \\
Total Escala Inteligencia Emocional & 5.30 & 0.78 \\
\hline \%FCM: \% Frecuencia Cardíaca Máxima; Media de puntos: Media de puntos anotados en los 3 \\
últimos partidos; Media de minutos: Media de minutos jugados en los 3 últimos partidos; ERP: \\
Escala de Rendimiento Percibido.
\end{tabular}

En la tabla 2 se muestran los resultados pertenecientes a las correlaciones bivariadas de las variables seleccionadas.

Sobre la base de estos resultados, se encuentra que la variable de carga interna, \%FCM, correlaciona positivamente con los valores obtenidos en la ERP (.391 y $p=.002$ ), por lo que a una mayor frecuencia cardíaca en entrenamiento los jugadores y jugadoras percibían un mayor rendimiento. Por parte de la variable de carga externa, $\mathrm{PL}$, se muestra una relación positiva con la fatiga (.285 y $p=.026)$, es decir, a mayor carga acumulada por los deportistas durante la sesión, implicará a una mayor percepción de fatiga previa a entrenamientos.

En cuanto a las variables Wellness, se encontró una relación positiva y fuerte entre ambas variables, Fatiga y Dolor M uscular (.755 y $p<.001)$. A su vez, se relacionaron negativamente con los Puntos, ERP, EEP y RE, es decir, a una mayor fatiga acumulada y, por tanto, Dolor Muscular, los jugadores anotaban menos puntos, percibían un rendimiento menor y su evaluación y regula ción de las emociones propias era negativa.

Las variables de rendimiento se correlacionaron positivamente entre sí (.543 y p<.001), disputar un 
mayor número de minutos durante el partido conlleva ba a un mayor número de puntos anotados. Por su parte, en cuanto a laERP ha correlacionado positivamente con todas las variables de IE. Las variables de IE correlacionaron sus resultados entre sí.

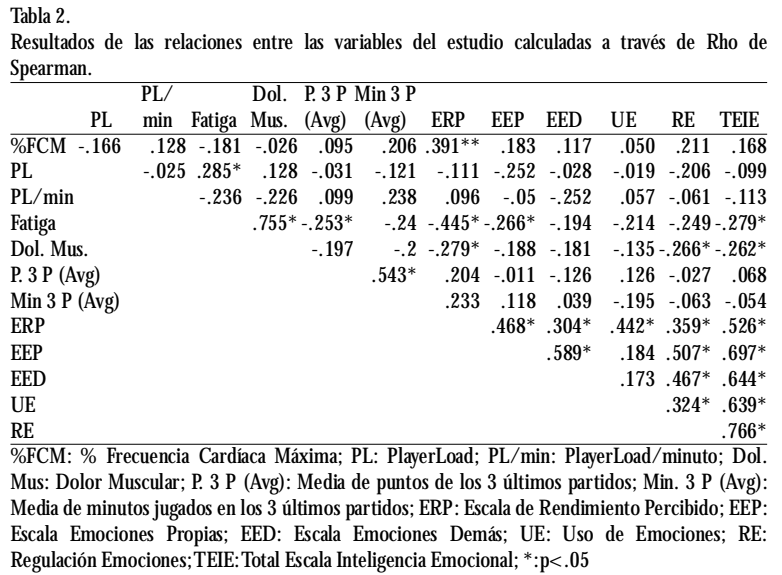
Media de minutos jugados en los 3 últimos partidos; ERP: Escala de Rendimiento Percibido; EEP Escala Emociones Propias; EED: Escala Emociones Demás; UE: Uso de Emociones; RE: Regulación Emociones; TEIE:Total Escala Inteligencia Emocional; *:p<.05

\section{Discusión}

Para una mejor comprensión de las variables del entrenamiento, el objetivo de este estudio fue identificar las relaciones entre la carga de entrenamiento que soportan los jugadores con variables relacionadas con el bienestar, la emoción y el rendimiento en baloncesto formativo. Esta es una de las primeras investigaciones que aúna de manera integral la carga de entrenamiento con aspectos emocionales. De esta forma, se va a facilitar a los entrenadores la gestión de esta información con el fin de planificar de forma más individualizada y específica las sesiones de entrenamiento. Con relación a las variables de carga, las respuestas internas más intensas se relacionaron de forma positiva con el rendimiento percibido por parte de los jugadores. Por otro lado, las demandas externas se relacionaron con valores ma yores de fatiga y, por tanto, dolor muscular. La fatiga y el dolor muscular influyeron en una menor percepción de rendimiento y de las emociones. Por su parte, el rendimiento percibido de los jugadores se relacionó positivamente con las variables referentes a la inteligencia emocional.

La monitorización de los entrenamientos, ya sea de forma objetiva o subjetiva, es de vital importancia a la hora de desarrollar contenidos y controlar la carga (Reina, et al. , 2019), tanto de manera interna como externa (Campos \& Toscano, 2018). El análisis de la carga externa e interna son métodos tradicionales utilizados para evaluar y entender las demandas físicas y fisiológicas en deportes de equipo ( 0 'G rady, Fox, Dalbo \& Scanlan, 2020). Se ha demostrado, que su combinación aporta información muy relevante a la hora de diferenciar las exigencias físicas y fisiológicas durante el entrenamiento y la competición en baloncesto (Stojanoviæ, et al., 2018). No obstante, en esta investigación, además de caracterizar cómo ha sido la carga durante el entrenamiento, se ha combinado con diversos factores como son el bienestar, el rendimiento o las emociones.

Los resultados de esta investigación han explicado que una mayor respuesta interna (FC) provocó en los deportistas una mejor evaluación de su rendimiento percibido. Los jugadores y jugadoras percibieron un mayor nivel en sus actuaciones cuando la actividad les resultaba más intensa. Sin embargo, por parte de la carga externa (PL acumulado), los resultados mostraron un aumento de la fatiga y dolor muscular en los jugadores previo al entrenamiento y, a su vez, estas últimas correlacionaron negativamente con el rendimiento percibido. Por ese motivo, es muy importante el diseño de tareas con el objetivo de que los jugadores puedan experimentar demandas similares a la competición (M ontgomery, Pyne \& M inahan, 2010). La modificación de los constreñimientos de las tareas repercute directamente sobre la carga que soportan los deportistas (Ibáñez, Pérez-Goye, García-Rubio \& Courel-Ibáñez, 2020). Los entrenadores de bal oncesto pueden manipular los ejercicios basados en los juegos de acuerdo con la fase estacional 0 el propósito de la sesión de entrena miento (O 'Grady, et al., 2020), y así, controlar la carga de forma progresiva, evitando que aparezca un aumento de la fatiga y, por tanto, sobreentrenamiento. En este sentido, varios factores modificables incluyen el tamaño del equipo (Conte, Favero, N iederhausen, Capranica \& Tessitore, 2017), áreas de juego (Atli, Köklü, Alemdaroglu \& Koçak, 2013), duración de juego y descanso (Conte, Favero, N iederhausen, Capranica \& Tessitore, 2016), reglas del juego (Conte, Favero, Niederhausen, Capranica\&Tessitore, 2015), tácticas (por ejemplo, juego ofensivo o defensivo) (Sansone, et al., 2019), y formaciones (Leite, Gonçal ves, Sampaio \& Saiz, 2013). Todo lo anterior se puede cambiar para manipular las cargas de trabajo experimentadas por los jugadores, generando una mayor respuesta interna y control de carga externa.

El bienestar puede ser evaluado en cada ámbito de lasalud: físico, emocional, social y espiritual (M CD owell, 2010). En este caso, se han descrito las variables de fatiga y dolor muscular. M onitorizar la presencia y la magnitud de la fatiga de los jugadores es necesario para evitar lesiones, pérdidas de rendimiento y un posible esta do de sobreentrenamiento (Twist \& Highton, 2013). 
McLean, et al. (2010), mostraron que la percepción de fatiga neuromuscular y dolor muscular se vieron afectados por el estrés del partido y, también, en función del contenido de entrenamiento dentro de cada microciclo. Esta herramienta ayuda a conocer la evolución de la fatiga en relación a la carga externa, incentivando a la creación de perfiles de recuperación (Campos \& Toscano, 2017). En este estudio, se ha encontrado una relación directa entre la carga acumulada durante el entrenamiento y la percepción de fatiga y dolor muscular por parte de los jugadores. Estos resultados van a permitir a los entrenadores, poder manipular la carga de entrenamiento con el objetivo de reducir la fatiga en los deportistas. El PlayerLoad es una herramienta objetiva y validada, a partir de la cual se puede indicar la tasa de estrés a la que está sometido el cuerpo del jugador durante un período de tiempo determinado (Barreira, et al., 2017). Ya se ha utilizado para evaluar la carga neuromuscular en diferentes deportistas con el fin de lograr una imagen más integrada y ecológica de las cargas de entrenamiento, pues se tra ta de una de las variables más predictivas del rendimiento (Schelling \& Torres, 2016). Poder correlacionar de forma positiva variables de carga externa objetiva con variables de fatiga percibida de forma subjetiva permite la posibilidad de monitorizar el entrenamiento sin grandes recursos económicos y materiales (Reina, et al., 2019), lo cual es un gran avance en el trabajo de recogida de datos durante el entrenamiento deportivo.

Con relación al rendimiento percibido, autorescomo Blanchard, M ask, Vallerand, de la Sablonnière and Provencher (2007) o Gillet, Berjot and Gobancé(2009), han demostrado una relación directa entre la motiva ción autodeterminada y el rendimiento deportivo. En el ámbito académico, se ha observado que aquellos estudiantes con una buena percepción de la competencia académica obtenían mejores resultados académicos (Gargallo, Garfella, Sanchez, Ros \& Serra, 2009). Algo similar ha ocurrido en el entrenamiento deportivo donde, aquellos deportistas con una mayor percepción de rendimiento se han relacionado con una mejor gestión de aspectos emocionales. En esta investigación se ha evaluado la inteligencia emocional de los deportistas ya que, se ha demostrado, que en el marco de la actividad física, se produce una relación directa entre las emociones y el bienestar de los deportistas (Núñez, León, González \& Martín-Albo, 2011; Romero, Zapata, García-Mas, Brustad, Garrido \& Letelier, 2010). Por este motivo, el interés acerca de la relación entre la inteligencia emocional con la salud se encuentra en con- tinuo crecimiento (Austin, Saklofske \& Egan, 2005). Estos datos corroboran la importancia de que los juga dores y jugadoras presenten un estado emocional positivo a la hora de rendir durante el entrenamiento deportivo. El concepto de inteligencia emocional se refiere a las diferencias individuales en la percepción, el procesamiento, la regulación y la utilización de la información emocional (Sáenz-López, Almagro \& Fernandez, 2017). Estas diferencias entre individuos han demostra do tener un impacto significativo sobre la salud física y mental (Extremera, Fernández-Berrocal \& Salovey, 2006). Por tanto, desarrollar una inteligencia emocional adecuada va a llevar a los deportistas a tomar decisiones más eficaces y, en consecuencia, un mayor rendimiento (Lane, 2007). En esta investigación se ha encontrado una relación directa entre el rendimiento percibido por los deportistas durante la competición con un buen uso de las emociones. Esto resulta esencial para la propiaadaptación físicay psicológicaal contexto social y deportivo (Mayer \& Salovey, 1997; Salovey, Mayer, Goldman, Turvey \& Palfai, 1995). Un desarrollo de la inteligencia emocional percibida provee al individuo de la capacidad de gestionar las emociones subjetivas cuando se enfrenta a situaciones estresantes (Salovey, et al., 2009), como las experimentadas durante la competición y el entrenamiento deportivo. Por tanto, un desarrollo correcto de la inteligencia emocional permitirá gestionar con éxito las situaciones negativas que se produzcan en el contexto deportivo, además de contar con estrategias de afrontamiento más adaptativas en la resolución de problemas sociales (Saklofske, Austin, Rohr \& Andrews, 2007). Por tanto, es importante su análisis y entendimiento dentro del ámbito del entrenamiento deportivo, como se ha ejecutado a lo largo de esta investigación. Siempre con el objetivo de conseguir un mayor rendimiento desde una perspectiva más global y bajo el nexo de diferentes vertientes sobre el análisis del entrenamiento, con el objetivo de dar una visión más integral de todas las capacidades del jugador de baloncesto.

Los resultados de esta investigación tienen una gran aplicabilidad al contexto deportivo, especialmente sobre la población estudiada. Será necesario incrementar la masa crítica de estudios para poder llegar a generalizar los hallazgos encontrados sobre la relación de las variables estudiadas.

\section{Conclusiones}

Gracias al uso exhaustivo de los diferentes factores 
que influyen de forma conjunta en el desarrollo integral de los jugadores y jugadoras de baloncesto en etapa formativa, los entrenadores van a aumentar sus recursos y conocimientos a la hora de planificar y diseñar las sesiones de entrenamiento. Esto se va a traducir en un aumento del rendimiento por parte de los deportistas, los cuales se desarrollarán de forma completa en todos los aspectos que tienen un factor condicionante dentro del ámbito deportivo.

Tres son las conclusiones que se alumbran tras el estudio de las relaciones entre la carga de entrenamiento y las emociones. El diseño de tareas que implican unas respuestas cardiacas más intensas va a provocar en los jugadores una mayor percepción de rendimiento en sus acciones, considerándola una práctica más eficaz. Por otro lado, un volumen alto de carga externa va a conllevar a una mayor fatiga percibida por los jugadores y, por ende, un mayor dolor muscular. Por tanto, es de vital importancia gestionar de forma adecuada la carga de entrenamiento, evitando unos mayores valores de fatiga previos a la competición deportiva. Finalmente, se puede concluir que el desarrollo de las emociones de manera estable va a condicionar también a una mejor percepción del rendimiento, es decir, mediante el desarrollo de tareas los entrenadores deben conseguir un clima emocional adecuado por parte de todos sus deportistas para que, en consecuencia, el rendimiento sea óptimo.

\section{Agradecimientos}

Estainvestigación hasido subvencionadaparcial mente por laAyuda a Grupos de Investigación (GR18170) de la Junta de Extremadura (Consejería de Economía e Infraestructuras); con la aportación de la U nión Europea a través de FEDER y para las ayudas económicas para estudiantes predoctorales que otorga la U niversidad de Extremadura a través de su Plan Propio de Investigación.

\section{Referencias}

Aguiar, M., Botelho, G., Lago, C., Maças, V., \& Sampaio, J. (2012). A review on the effects of soccer small-sided games. Journal of H uman Kinetics, 33, 103-113. https: / I doi.org/ 10.2478/ v10078-012-0049-x

Atli, H., Köklü,Y.,Alemdaroglu, U ., \& Koçak, F. Ü. (2013).A comparison of heart rate response and frequencies of technical actions between half-court and full-court 3-aside games in high school female basketball players. The
Journal of Strength and Conditioning Research, $27(2), 352-$ 356 . https: / / do i . org/ 10.1519/ JSC.0b013e3182542674

Austin, E. J., Saklofske, D. H. , \& Egan, V. (2005). Personality, well-being and health correlates of trait emotional intelligence. Personality Individual Differences 38(3), 547558. https: / / doi.org/ 10.1016/ j. paid. 2004.05.009

Barreira, P., Robinson, M. A. , Drust, B., N edergaard, N., Raja Azidin, R. M. F., \&Vanrenterghem, J. (2017). M echanical Player Load ${ }^{\mathrm{TM}}$ using trunk-mounted accelerometry in football: Is it a reliable, task-and player-specific observation? Journal of SportsSciences35(17), 1674-1681. https: / / doi.org/ 10.1080/ 02640414.2016.1229015

BastidaC astillo, A., Gómez-Carmona, C. D., laCruz-Sánchez, D., Reche-Royo, X. , Ibáñez, S. J., \& Pino 0 rtega, J. J. A. S. (2019). Accuracy and inter-unit reliability of ultra-wideband tracking system in indoor exercise. Applied Sciences, 9(5), 939. https: / / doi.org/ 10.3390/ app9050939

Blanchard, C. M., M ask, L., Vallerand, R. J., delaSablonnière, R., \& Provencher, P. (2007). Reciprocal relationships between contextual and situational motivation in a sport setting. Psychology of Sport Exercise 8(5), 854-873. https:/ / doi.org/ 10.1016/ j.psychsport. 2007.03.004

Borg, G. (1962). A simplerating scal efor usein physical work test. Håkan 0 hlssons boktryckeri.

Boyd, L. J., Ball, K., Aughey, R.J. (2011). The reliability of M inimaxX accelerometersfor measuring physical activity inAustralian football. International Journal of SportsPhysiology, 6(3), 311-321. https:/ / doi.org/ 10.1123/ ijspp. 6.3.311

Buchheit, M. , Racinais, S., Bilsborough, J., Bourdon, P., Voss, S., Hocking, J.,... Coutts, A. (2013). M onitoring fitness, fatigue and running performance during a pre-season training camp in elite football players. Journal of Science Medicine in Sport 16(6), 550-555. https:/ / doi.org/ 10.1016/ j.jsams. 2012.12.003

Campos, M. \& \&Toscano, F. (2017). M onitorización delacarga deentrenamiento, lacondición física, lafatigay el rendimiento duranteel microcicl o competitivo en fútbol. Re vista de Preparación Física en el Fútbol, 5050.

Campos, M. A., \& Toscano, F. J. (2018). Comparación de la percepción subjetivadel esfuerzo entre partidos amistososy diferentestipos de sesión en futbolistas profesionales. Retos: nuevastendencias en educación física, deportey recreación, (34), 66-70.

Castagna, C., Impellizzeri, F. M., Chaouachi,A., Bordon, C., \& Manzi,V. (2011). Effect of training intensity distribution on aerobic fitness variables in elite soccer players: acase study. The Journal of Strength and Conditioning Research 25(1), 66-71. doi:10.1519/ JSC.0b013e3181fef3d3

Chambers, R., Gabbett,T. J., Cole, M. H., \& Beard,A. (2015). The use of wearable microsensors to quantify sportspecific movements. Sports M edicine, 45 (7), 1065-1081. Conte, D., Favero, T., N iederhausen, M., Capranica, L., \& 
Tessitore,A. (2017). Effect of number of playersand maturity on ball-drillstraining load in youth basketball. Sports, 5(1), 3. https./ / doi.org/ 10.3390/ sports5010003

Conte, D., Favero,T.G., Niederhausen, M., Capranica, L., \&Tessitore, A. (2015). Physiological and technical demands of no dribble game drill in young basketball players The Journal of Strength and Conditioning Research, 29(12), 3375-3379. doi:10.1519/ ISC.0000000000000997

Conte, D., Favero,T.G., Niederhausen, M.,Capranica, L., \&Tessitore, A. (2016). Effect of different number of playersand training regimeson physiological and technical demands of ball-drills in basketball. Journal of SportsSciences, 34(8), 780-786. https./ / doi.org/ 10.1080/ 02640414.2015.1069384

Declaración de Helsinki delaAMM (2013). Principioséticos para lasinvestigacionesmédicasen sereshumanos (64AsambleaGenera). Fortaleza, Brasil.

Extremera, N., Fernández-Berroca, P., \& Salovey, P. (2006). Spanish version of theM ayer-Salovey-Caruso Emotional Intelligence Test (MSCEIT). Version 2.0: reliabilities, age and gender differences. Psicothema, 18, 42-48.

Fox, J., Scanlan, A., \& Stanton, R. (2017). A review of player monitoringapproachesinbasketball: Currenttrendsandfuture directions The Journal of Strength Conditioning \& Research 31(7), 2021-2029. doi:10.1519/JSC.0000000000001964

Gargallo, B., Garfella, P. R., Sanchez, F., Ros, C., \& Serra, B. (2009). Lainfluenciadel autoconcepto en el rendimiento académico en estudiantesuniversitarios. Revista Española deO rientación y Psicopedagogía, 20(1), 16-28. http:/ / hdl. handle. net/ 20.500.12466/ 1127

Gillet, N., Berjot, S., \& Gobancé, L. (2009).A motivational model of performancein thesport domain. European Journal of Sport Science, 9(3), 151-158. https:/ / doi.org/ 10.1080/ 17461390902736793

Hernández, D., Casamichana, D. \& Sánchez-Sánchez,J. (2017). La cuantificación delacargadeentrenamiento como estrategia básicadeprevención delesiones Revista dePreparación Físca en el Fútbol, 24, 33-39.

Ibáñez,S. J.,Antúnez,A.,Pino-O rtega,J., \& Garcíar Rubio,J. (2018). Control del entrenamiento medianteel empleo detecnologíasen tiempo real en balonmano. EnS. Feu, J. GarcíaRubio, yS. J. Ibáñez(Eds), Avancescientíficospara el aprendizajey desarrollode balonmano (pp. 167-192). Cáceres: Servicio dePublicacionesdeU niversidad deExtremadura.

Ibáñez, S. J., García Rubio,J., Gómez, M.-Á., \& González-Espino$\mathrm{sa}, \mathrm{S}$. (2018). The impact of rule modifications on elite basketball teams' performance. Journal of H uman Kinetics, 64(1), 181-193. https./ / doi.org/ 10.1515/ hukin-2017-0193

Ibáñez, S. J., Garcia-Rubio, J., Rodríguez-Serrano, D., \& Feu, S. (2019). Development of a Knockout Competition in Basketball: A Study of the Spanish Copa del Rey. Frontiers in Psychology, 10. https. / doi.org/ 10.3389/ fpsyg2019.02457 Ibáñez, S. J., Pérez-Goye, E., Garcia Rubio,J., \& Courel-Ibáñez, J. (2020). Effectsof task constraintson trainingworkload in elite women'ssoccer. International Journal of SportsScience\& Coaching, 15(1), 99-107. http:// dx.doi.org/ 10.1177/ 1747954119891158.

Kais, K. \& \& Raudsepp, L. (2004). Cognitiveand somatic anxiety and self-confidencein athletic performance of beach volleybal. Perceptual Motor Skills 98(2), 439-449. https:/ / doi.org/ 10.2466\%2Fpms. 98.2.439-449

Lane, A. M. (2007). Mood and human performance Conceptual, Measurement, and Applied Issues. NewYork: N ovaPublishers.

Leite, N. M. C., Gonçalves, B. S.V., Sampaio,A.J. D. E., \& Sàz, S. J. (2013). Effectsof the playing formation and gameformat on heart rate, rateof perceived exertion, vertical jump, individual and collective performance indicators in youth basketball training International SportM ed Journal, 14(3), 127-138. https:/ / hdl. handle. net/ 10520/ EJC142369

Lourenço, J.,Almagro, B. . . \& Sáenz-López, P. (2018).Validación del Cuestionario de Rendimiento Percibido en el Deporte. Ebalonmano. com: Revista deCienciasdel Deporte, 14(3), 195-204. http:/ / hdl. handle. net/ 10662/ 8684

Mas, J. R. L., Guzmán,J. F., \& Martínez, R. (2018). Comparación delafrecuenciacardiacaen competición, entrejugadoresde pádel deelitey decategoríanacional. Retos nuevastendenciasen educación física, deportey recreación, 1(33), 91-95.

M ayer, J. D. , \& Salovey, P. (1997).W hat isemotional intelligence? En P. Salovey y D. Sluyter (Eds), Emotional Development and Emotional IntelligenceI Implicationsfor Educators(pp. 3-31). Nueva York: BasicBooks.

McD owell, I. (2010). Measures of self-perceived well-being. Journal of Psychosomatic Rescarch, 69(1), 69-79. doi: 10.1016/ j.jpsychores. 2009.07.002.

McGarry,T. (2009). Applied and theoretical perspectives of performance analysis in sport: Scientific issues and challenges International Journal of PerformanceAnalysisin Sport, 9 (1), 128140. https./ / doi.org/ 10.1080/ 24748668.2009.11868469

McLean, B.D., Coutts,A.J., Kelly,V., McGuigan, M. R., \& Cormack, S. J. (2010). Neuromuscular, endocrine, and perceptual fatigue responses during different length between-match microcyclesin professional rugby leagueplayers. International Journal of SportsPhysiology Performance5(3), 367-383. https:/ / doi.org/ 10.1123/ ijspp.5.3.367

Mercadet-Portillo, O. E. \& Inufio-Díaz, R. (2015). Inteligencia emocional y el enfado en baloncesto. Revista científica especializada en Cultura Físca y Deportes, 12 (26), 111-122.

Meyer, B. B. \& \& Fletcher,T. B. (2007). Emotional intelligence: A theoretical overview and implications for research and professional practice in sport psychology. Journal of Applied Sport Psychology, 19(1), 1-15. https:/ / doi.org/ 10.1080/ 10413200601102904

Miao, C., Humphrey, R. H., \& Qian, S. J.P. (2017).A metazanalysis of emotional intelligence and its relationships with organiztional citizenshipbehavior and counterproductivework behavior. Personality Individual Differences116, 144-156. https: / doi.org/ 10.1016/ j.paid.2017.04.015 
M ontgomery, P. G., Pyne, D. B. , \& M inahan, C. L. (2010).The physical and physiological demands of basketball trainingand competition. International Journal of SportsPhysiology Performance 5(1), 75-86. https:/ / doi.org/ 10.1123/ ijspp.5.1.75

Ng, K.-M.,Wang, C., Zalaquett, C. P., \& Bodenhorn, N. (2008).A confirmatory factor analysis of theW ongand Law Emotional IntelligenceScalein asampleof international collegestudents. International Journal for the Advancement of Counselling, 30(2), 131-144. DOI:10.1007/ s10447-007-9037-6

Núñez, J. L., León, J., González, V., \& Martín-Albo, J. (2011). Propuestadeun modelo explicativo del bienestar psicológico en el contexto deportivo. Revista dePsicología del Deporte,20(1), 223-242.

O 'Grady, C. J., Fox, J. L., Dalbo, V. ., \& Scanlan,A.T. (2020). A Systematic Review of the External and Internal Workloads ExperiencedDuring Games-Based Drillsin Basketball Players International Journal of SportsPhysiology Peformance15(5), 603616. https: / doi.org/ 10.1123/ ijspp.2019-0785

Petway,A.J., Freitas,T.T., Calleja_González,J., M edinaLeal, D., \& Alcaraz, P. E. (2020). Trainingload and match-playdemandsin basketball based on competition level: A systematic review. PloS one, 15(3), e0229212. https:/ / doi.org/ 10.1371/ journal. pone. 0229212

Pacheco, N. E., Rey, L., \& Sánchez-Álvarez, N. (2019).Validation of the Spanish version of theWong Law emotional intelligence scale(W LEIS-S). Psicothema, 31(1), 94-100.

Reina, M., Rubio,J.G.,Antúnez,A., \& Ibáñez, S. J. (2020). Compz ración delacargainternay externaen competición oficial de3 vs. $3 y 5$ vs. 5 en baloncesto femenino. Retos,37(37), 400-405.

Reina, M., GarcíaRubio, J., \& Ibáñez, S. J. (2020). Training and Competition Load inFemaleBasketball:A Systematic Review. International Journal of Environmental Research PublicH ealth 17(8), 2639. https:/ / doi.org/ 10.3390/ ijerph17082639

Reina, M., Mancha Triguero, D., García-Santos, D., GarcíaRubio, J., \& Ibáñez, S. J. (2019). Comparación de tres métodos de cuantificación delacargadeentrenamiento en baloncesto. Re vista Internacional de Ciencias de Deporte, 15(58), 368-382. https:/ / doi.org/ 10.5232/ ricyde2019.05805

Romero,A.E.,Zapđa, R.Z., GarcíaMas,A., Brustad, R.J., Garrido, R., \& Letelier, A. (2010). Estrategias de afrontamiento y bienestar psicológico en jóvenestenistasdecompetición. Re vista dePsicología del Deporte, 19(1), 117-133.

Sáenz-López, P.,Almagro, B., \& Fernández, E. J. (2017). M otivación, Inteligencia emocional y actividad físca en universitarios (Vol. 24). Huelva: Servicio dePublicacionesdelaU niversidad deH Helva.

Sáenz-López, P., Ibáñez, S., Giménez, J., Sierra,A., \& Sánchez, M. (2005). Multifactor characteristics in the process of development of the male expert basketball player in Spain. International Journal of Sport Psychology, 36(2), 151-171.

Szklofske, D. H.,Austin, E. J., Rohr, B.A., \&Andrews, J. (2007). Personality, emotiona intelligenceand exercise Journal of $H$ ealth Psychology, 12(6), 937-948. https:// doi.org/ $10.1177 \% 2 F 1359105307082458$
Salovey, P., Mayer, J. D., Caruso, D. \&YOo, S. H. (2009).Thepositive psychology of emotional intelligence. InS. J. L. \& C. R. Snyder (Ed.), Thehandbook of positivepsychology (pp. 237-248). Nueva York: 0 xford U niversity Press

Salovey, P., Mayer, J. D., Goldman, S. L.,Turvey, C., \& Palfai,T. P. (1995). Emotional attention, clarity, and repar: Exploring emotiona intelligenceusing theTraitM eta-M ood Scale. In]. W. Pennebaker (Ed.), Emotion, disclosure and health (pp. 125154).Washington:American PsychologicalAssociation. https:/ / psycnet. apa.org/ doi/ 10.1037/ 10182-006

Sansone, P., Tessitore, A., Paulauskas, H., Lukonaitiene, I., Tschan, H., Pliauga, V. \& C Conte, D. (2019). Physical and physiological demands and hormonal responses in basketball small-sided gameswith different tactical tasksand training regimes J ournal of Science M edicinein Sport 22(5), 602-606. https: / / doi.org/ 10.1016/ j.jsams.2018.11.017

Schelling, X. \& \&Torres, L. (2016). Accelerometer load profilesfor basketball-specificdrillsin eliteplayers. Journal of SportsScience Medicine 15(4), 5855-591.

Stojanoviæ, E., Stojlijkoviæ, N., Scanlan, A. T., Dalbo, V. J., Berkelmans, D. M., \& Milanoviæ, Z. (2018). The activity demands and physiological responses encountered during basketball match-play: asystematic review. Journal SportsM edicine48(1), 111-135.

Thomas, J. R., Nelson, J. K. \& Silverman, S. J. (2015). Research methods in physical activity: Human kinetics. United States of America

Twist, C., \& Highton,J. (2013). Monitoringfatigueand recoveryin rugby league players International Journal of Sports Physiology Performance 8(5), 467-474. https:/ / doi.org/ 10.1123/ ijspp.8.5.467

U phill, M., Groom, R., yJones, M. (2014). Theinfluence of ingameemotionson bakketball performance. European J ournal of Sport Science, 14(1), 76-83. https:/ / doi.org/ 10.1080/ 17461391.2012 .729088

Wong, C.S., \& Law, K. S. (2002). Theeffectsof leader and follower emotional intelligence on performance and attitude: $A n$ exploratory study. The Leadership Quarterly, 13(3), 243-274. https: / / doi.org/ 10.1016/ S1048-9843(02)00099-1

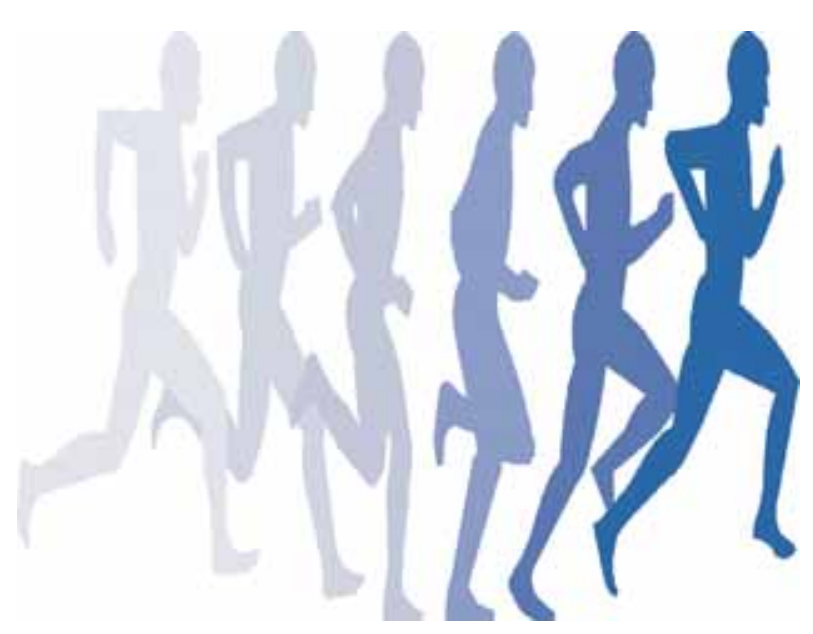

